\title{
Variational Approach to Impulsive Problems: A Survey of Recent Results
}

\author{
Fang-fang Liao ${ }^{1}$ and Juntao Sun ${ }^{2}$ \\ ${ }^{1}$ Nanjing College of Information Technology, Nanjing 210046, China \\ ${ }^{2}$ School of Science, Shandong University of Technology, Zibo 255049, China \\ Correspondence should be addressed to Juntao Sun; sunjuntao2008@163.com
}

Received 21 April 2014; Accepted 5 May 2014; Published 20 May 2014

Academic Editor: Yonghui Xia

Copyright (c) 2014 F.-f. Liao and J. Sun. This is an open access article distributed under the Creative Commons Attribution License, which permits unrestricted use, distribution, and reproduction in any medium, provided the original work is properly cited.

We present a survey on the existence of nontrivial solutions to impulsive differential equations by using variational methods, including solutions to boundary value problems, periodic solutions, and homoclinic solutions.

\section{Introduction}

There are many processes and phenomena in the real world, which are subjected during their development to the shortterm external influences. Their duration is negligible compared with the total duration of the studied phenomena and processes. Therefore, it can be assumed that these external effects are "instantaneous"; that is, they are in the form of impulses. The investigation of such "leaps and bounds" developing dynamical states is a subject of different sciences: mechanics, control theory, pharmacokinetics, epidemiology, population dynamics, economics, ecology, and so forth. We refer the reader to [1-6].

During the last two decades, the theory of the existence of solutions for impulsive differential equations has attracted much attention because of its important applications; see [3, 7-12]. Classical approaches to such problems include fixed point theory and the method of upper and lower solutions. In 2008, Tian and Ge first applied variational methods in the study of boundary value problems (BVPs) of impulsive differential equations. From then on, variational methods have been widely used to study impulsive problems, such as boundary value problems, periodic solutions, and homoclinic solutions. We refer the reader to [3, 13-22].

The main aim of this survey is to present some recent existence results for impulsive differential equations via variational methods. More precisely, we will explore the variational framework of impulsive differential equations and study the existence and multiplicity of solutions of boundary value problems, periodic solutions, and homoclinic solutions.

The rest of this paper is organized as follows. In Section 2, we will state some important results for the second-order impulsive differential equations with two kinds of boundary conditions. Periodic solutions to impulsive problems will be considered in Section 3. In Section 4, homoclinic solutions to impulsive differential equations will be studied.

Finally in this section, we must note that besides the results presented in this survey many interesting and important results on impulsive differential equations via variational methods have been obtained by other researchers; see, for example, [23-31] and the references cited therein.

\section{Boundary Value Problems}

In this section, we recall some results of boundary value problems for impulsive differential equations.

Firstly, we consider a class of impulsive problems with Dirichlet boundary condition

$$
\begin{gathered}
-u^{\prime \prime}(t)+\lambda u(t)=f(t, u(t))+g(t), \quad \text { a.e. } t \in[0, T], \\
\Delta u^{\prime}\left(t_{j}\right)=u^{\prime}\left(t_{j}^{+}\right)-u^{\prime}\left(t_{j}^{-}\right)=I_{j}\left(u\left(t_{j}\right)\right), \quad j=1,2, \ldots, p, \\
u(0)=u(T)=0,
\end{gathered}
$$


where $\lambda$ is a constant, $f:[0, T] \times \mathbb{R} \rightarrow \mathbb{R}$ is continuous, $g \in L^{2}[0, T]$ and $I_{j}: \mathbb{R} \rightarrow \mathbb{R}, j=1,2, \ldots, p$, are continuous.

From now on, we assume that $\lambda>-\left(\pi^{2} / T^{2}\right)$ and define

$$
\begin{array}{r}
L(u, u)=\frac{1}{2} \int_{0}^{T}\left|u^{\prime}(t)\right|^{2} d t+\frac{\lambda}{2} \int_{0}^{T}|u(t)|^{2} d t \\
\text { for any } u \in H_{0}^{1}(0, T) .
\end{array}
$$

Then we have the following.

Proposition 1 (see [21, Proposition 1.1]). If $\lambda>-\left(\pi^{2} / T^{2}\right)$, then there exist constants $b>a>0$ such that

$$
a\|u\|_{H_{0}^{1}}^{2} \leq L(u, u) \leq b\|u\|_{H_{0}^{1}}^{2}, \quad \text { for any } u \in H_{0}^{1}(0, T) .
$$

Definition 2. One says that a function $u \in H_{0}^{1}(0, T)$ is a weak solution to problem (1) if

$$
\begin{gathered}
\int_{0}^{T}\left(u^{\prime}(t), v^{\prime}(t)\right)+\lambda(u(t), v(t)) d t+\sum_{j=1}^{p} I_{j}\left(u\left(t_{j}\right)\right) v\left(t_{j}\right) \\
=\int_{0}^{T} f(t, u) v d t+\int_{0}^{T} g(t) v d t
\end{gathered}
$$

holds for any $v \in H_{0}^{1}(0, T)$.

Theorem 3 (see [15, Theorem 4.2]). Suppose that $g \equiv 0, f$ is bounded, and the impulsive functions $I_{j}$ are bounded. Then problem (1) has at least one weak solution to $\lambda>-\left(\pi^{2} / T^{2}\right)$.

If $f$ is sublinear at infinity on $u$ and the impulsive functions $I_{j}$ have sublinear growth, then we have the following.

Theorem 4 (see [15, Theorem 4.3]). Assume that $g \equiv 0$ and the following conditions are satisfied.

(F1) There exist $a, b>0$ and $\gamma \in(0,1)$ such that

$|f(t, u)| \leq a+b|u|^{\gamma}, \quad$ for every $(t, u) \in[0, T] \times \mathbb{R}$

(I1) there exist $a_{j}, b_{j}>0$ and $\gamma_{j} \in[0,1)(j=1,2, \ldots, p)$ such that

$\left|I_{j}(u)\right| \leq a_{j}+b_{j}|u|^{\gamma_{j}}, \quad$ for every $u \in \mathbb{R}(j=1,2, \ldots, p)$.

Then problem (1) has at least one weak solution to $\lambda>$ $-\left(\pi^{2} / T^{2}\right)$.

If $f$ is superlinear at infinity on $u$, then we have the following.

Theorem 5 (see [21, Theorem 1.2]). Assume that (I1) holds and the following conditions are satisfied.

(F2) There is a constant $\mu>2$ such that $0<\mu F(t, u) \leq$ $u f(t, u)$ for every $t \in[0, T]$ and $u \in \mathbb{R} \backslash\{0\}$;
(G) $g \in L^{2}[0, T]$ satisfies the following inequality

$$
a-\frac{M}{\lambda_{1}}-\sum_{j=1}^{p} T\left(a_{j}+b_{j}\right)-\frac{\sqrt{T}\|g\|_{L^{2}}}{\sqrt{\lambda_{1}}}>0,
$$

where $M:=\sup \{F(t, u): t \in[0, T],|u|=1\}$ and $a$ is defined in Proposition 1.

Then problem (1) has at least one weak solution to $\lambda>$ $-\left(\pi^{2} / T^{2}\right)$.

Furthermore, we have the following result of multiple solutions.

Theorem 6 (see [21, Theorem 1.3]). Let $g(t) \equiv 0$. Assume that the conditions (F2), (I1), and (G) hold. Moreover, $f$ and the impulsive functions $I_{j}$ are odd about $u$; then problem (1) has infinitely many solutions.

Next, we consider another Dirichlet impulsive problem

$$
\begin{gathered}
-u^{\prime \prime}(t)+q(t) u(t)=f(t, u(t)), \quad \text { a.e. } t \in[0, T], \\
\Delta u^{\prime}\left(t_{j}\right)=u^{\prime}\left(t_{j}^{+}\right)-u^{\prime}\left(t_{j}^{-}\right)=I_{j}\left(u\left(t_{j}\right)\right), \quad j=1,2, \ldots, p, \\
u(0)=u(T)=0,
\end{gathered}
$$

where $q \in L^{\infty}[0, T], f:[0, T] \times \mathbb{R} \rightarrow \mathbb{R}$ is continuous, and $I_{j}: \mathbb{R} \rightarrow \mathbb{R}, j=1,2, \ldots, p$, are continuous.

Theorem 7 (see [20, Theorem 3.1]). Let $d_{j}(j=1,2 \ldots, p)$ be fixed constants. If $f(t, u)=\sigma(t) \in L^{2}(0, T)$ and $I_{j}(u)=$ $d_{j}(j=1,2, \ldots, p)$; then problem (8) has a unique solution $u$ if ess $\inf _{t \in[0, T]} q(t)>-\left(\pi^{2} / T^{2}\right)$.

Similar to Theorem 4, we have the following.

Theorem 8 (see [20, Theorem 3.2]). Assume that conditions (F1) and (I1) hold. Then problem (8) has at least one weak solution to ess inf $\operatorname{in}_{t \in[, T]} q(t)>-\left(\pi^{2} / T^{2}\right)$.

If condition $(I 1)$ is replaced by the following condition:

(I3) $I_{j}(j=1,2 \ldots, p)$ satisfy $\int_{0}^{u} I_{j}(s) d s \geq 0$ for all $u \in \mathbb{R}$, then we have the following.

Theorem 9 (see [30, Theorem 3.1]). Assume that conditions (F1) and (I3) hold. Then problem (8) has at least one weak solution to ess $\inf _{t \in[0, T]} q(t)>-\left(\pi^{2} / T^{2}\right)$.

Now we study a class of impulsive Hamiltonian systems with periodic boundary conditions

$$
\begin{gathered}
-\ddot{u}+A(t) u=\lambda \nabla F(t, u)+\mu \nabla G(t, u), \quad \text { a.e. } t \in[0, T], \\
\Delta\left(\dot{u}^{i}\left(t_{j}\right)\right)=\dot{u}^{i}\left(t_{j}^{+}\right)-\dot{u}^{i}\left(t_{j}^{-}\right)=I_{i j}\left(u^{i}\left(t_{j}\right)\right), \\
i=1,2, \ldots, N, \quad j=1,2, \ldots, l, \\
u(0)-u(T)=\dot{u}(0)-\dot{u}(T)=0,
\end{gathered}
$$


where $A:[0, T] \rightarrow \mathbb{R}^{N \times N}$ is a continuous map from the interval $[0, T]$ to the set of $N$-order symmetric matrices, $\lambda$, $\mu$ are two positive parameters, $T$ is a real positive number, $u(t)=\left(u^{1}(t), u^{2}(t), \ldots, u^{N}(t)\right), t_{j}, j=1,2, \ldots, l$, are the instants where the impulses occur and $0=t_{0}<t_{1}<t_{2}<$ $\cdots<t_{l}<t_{l+1}=T, I_{i j}: \mathbb{R} \rightarrow \mathbb{R}(i=1,2, \ldots, N, j=$ $1,2, \ldots, l)$ are continuous, and $F, G:[0, T] \times \mathbb{R}^{N} \rightarrow \mathbb{R}$ are measurable with respect to $t$, for every $u \in \mathbb{R}^{N}$, continuously differentiable in $u$, for almost every $t \in[0, T]$, and satisfy the following standard summability condition:

$$
\begin{aligned}
& \sup _{|u| \leq b} \max \{|F(\cdot, u)|,|G(\cdot, u)|,|\nabla F(\cdot, u)|, \\
&|\nabla G(\cdot, u)|\} \in L^{1}(0, T), \quad \forall b>0 .
\end{aligned}
$$

If $X$ is a real Banach space, denote by $\mathscr{W}_{X}$ the class of all functionals $\Phi: X \rightarrow \mathbb{R}$ possessing the following property: if $\left\{u_{n}\right\}$ is a sequence in $X$ converging weakly to $u \in X$ and $\liminf _{n \rightarrow \infty} \Phi\left(u_{n}\right) \leq \Phi(u)$, then $\left\{u_{n}\right\}$ has a subsequence converging strongly to $u$.

For example, if $X$ is uniformly convex and $g:[0,+\infty) \rightarrow$ $\mathbb{R}$ is a continuous, strictly increasing function, then, by a classical result, the functional $u \rightarrow g(\|u\|)$ belongs to the class $\mathscr{W}_{X}$.

Theorem 10 (see [32]). Let $X$ be a separable and reflexive real Banach space; let $\Phi: X \rightarrow \mathbb{R}$ be a coercive, sequentially weakly lower semicontinuous $C^{1}$ functional, belonging to $\mathscr{W}_{X}$, bounded on each bounded subset of $X$, whose derivative admits a continuous inverse on $X^{*} ; J: X \rightarrow \mathbb{R}$ is a $C^{1}$ functional with compact derivative. Assume that $\Phi$ has a strict local minimum $u_{0}$ with $\Phi\left(u_{0}\right)=J\left(u_{0}\right)=0$. Finally, setting

$$
\begin{gathered}
\alpha=\max \left\{0, \limsup _{\|u\| \rightarrow+\infty} \frac{J(u)}{\Phi(u)}, \limsup _{u \rightarrow u_{0}} \frac{J(u)}{\Phi(u)}\right\}, \\
\beta=\sup _{u \in \Phi^{-1}((0,+\infty))} \frac{J(u)}{\Phi(u)},
\end{gathered}
$$

assume that $\alpha<\beta$.

Then, for each compact interval $[a, b] \subset(1 / \beta, 1 / \alpha)$ (with the conventions $1 / 0=+\infty, 1 /+\infty=0)$, there exists $R>0$ with the following property: for every $\lambda \in[a, b]$ and every $C^{1}$ functional $\Psi: X \rightarrow \mathbb{R}$ with compact derivative, there exists $\delta>0$ such that, for each $\mu \in[0, \delta]$,

$$
\Phi^{\prime}(x)=\lambda J^{\prime}(x)+\mu \Psi^{\prime}(x)
$$

has at least three solutions in $X$ whose norms are less than $R$.

The following two results of Ricceri guarantee the existence of three solutions for a given equation.

Theorem 11 (see [33]). Let $X$ be a reflexive real Banach space; $I \subseteq \mathbb{R}$ is an interval; let $\Phi: X \rightarrow \mathbb{R}$ be a sequentially weakly lower semicontinuous $C^{1}$ functional, bounded on each bounded subset of $X$, whose derivative admits a continuous inverse on $X^{*} ;-J: X \rightarrow \mathbb{R}$ is a $C^{1}$ functional with compact derivative. Assume that

$$
\lim _{\|x\| \rightarrow+\infty}(\Phi(x)-\lambda J(x))=+\infty
$$

for all $\lambda \in I$ and that there exists $\rho \in \mathbb{R}$ such that

$$
\begin{aligned}
\operatorname{supinf}_{\lambda \in I}(\Phi \in X & (x)+\lambda(\rho-J(x))) \\
& <\inf _{x \in X} \sup _{\lambda \in I}(\Phi(x)+\lambda(\rho-J(x))) .
\end{aligned}
$$

Then there exist a nonempty open set $A \subseteq I$ and a positive number $R$ with the following property: for every $\lambda \in A$ and every $C^{1}$ functional $-\Psi: X \rightarrow \mathbb{R}$ with compact derivative, there exists $\delta>0$ such that, for each $\mu \in[0, \delta]$,

$$
\Phi^{\prime}(x)-\lambda J^{\prime}(x)-\mu \Psi^{\prime}(x)=0
$$

has at least three solutions in $X$ whose norms are less than $R$.

Proposition 12 (see [34]). Let $X$ be a nonempty set and $\Phi, J$ two real functions on $X$. Assume that there are $r>0$ and $x_{0}, x_{1} \in X$ such that

$$
\begin{gathered}
\Phi\left(x_{0}\right)=J\left(x_{0}\right)=0, \quad \Phi\left(x_{1}\right)>r, \\
\sup _{x \in \Phi^{-1}((-\infty, r])} J(x)<r \frac{J\left(x_{1}\right)}{\Phi\left(x_{1}\right)} .
\end{gathered}
$$

Then, for each $\rho$, satisfying

$$
\sup _{x \in \Phi^{-1}((-\infty, r])} J(x)<\rho<r \frac{J\left(x_{1}\right)}{\Phi\left(x_{1}\right)},
$$

one has

$$
\begin{aligned}
& \operatorname{supinf}_{\lambda \geq 0}(\Phi(x)+\lambda(\rho-J(x))) \\
&<\inf _{x \in X} \sup _{\lambda \geq 0}(\Phi(x)+\lambda(\rho-J(x))) .
\end{aligned}
$$

We always assume that the following conditions hold:

$\left(\mathscr{A}_{1}\right) A(t)=\left(a_{i j}(t)\right)$ is a symmetric matrix with $a_{i j} \in$ $L^{\infty}([0, T])$ for every $t \in[0, T]$;

$\left(\mathscr{A}_{2}\right)$ there exists a positive constant $v$ such that

$$
A(t) u \cdot u \geq v|u|^{2}, \quad \text { for every } u \in \mathbb{R}^{N} \text {, a.e. } t \in[0, T] \text {; }
$$

$\left(\mathscr{I}_{1}\right)$ for any $i=1,2, \ldots, N$, and $j=1,2, \ldots, l, I_{i j}(t)$ are nondecreasing in $t \in \mathbb{R}$ and

$$
I_{i j}(t) t \geq 0, \quad \forall t \in \mathbb{R} .
$$

Define the Sobolev space by

$$
\begin{gathered}
H_{T}^{1}:=\left\{u:[0, T] \longrightarrow \mathbb{R}^{N}: u\right. \text { is absolutely continuous, } \\
\left.u(0)=u(T), \text { and } \dot{u} \in L^{2}(0, T)\right\}
\end{gathered}
$$


with the inner product

$$
\begin{array}{r}
\langle u, v\rangle=\int_{0}^{T}(u(t), v(t)) d t+\int_{0}^{T}(\dot{u}(t), \dot{v}(t)) d t, \\
\forall u, v \in H_{T}^{1},
\end{array}
$$

where $(\cdot, \cdot)$ denotes the inner product in $\mathbb{R}^{N}$. The corresponding norm is defined by

$$
\|u\|_{H_{T}^{1}}=\left(\int_{0}^{T}|u(t)|^{2} d t+\int_{0}^{T}|\dot{u}(t)|^{2} d t\right)^{1 / 2}, \quad \forall u \in H_{T}^{1} .
$$

For every $u, v \in H_{T}^{1}$, we define

$$
\begin{aligned}
\langle u, v\rangle_{1}= & \int_{0}^{T}(A(t) u(t), v(t)) d t \\
& +\int_{0}^{T}(\dot{u}(t), \dot{v}(t)) d t, \quad \forall u, v \in H_{T}^{1}
\end{aligned}
$$

and observe that, using assumptions $\left(\mathscr{A}_{1}\right)$ and $\left(\mathscr{A}_{2}\right),(24)$ defines an inner product in $H_{T}^{1}$, whose corresponding norm is

$$
\|u\|:=\langle u, u\rangle_{1}^{1 / 2}
$$

A simple computation shows that

$$
(A(t) u, u)=\sum_{i, j=1}^{N} a_{i, j}(t) u^{i} u^{j} \leq \sum_{i, j=1}^{N}\left\|a_{i, j}\right\|_{\infty}|u|^{2},
$$

for every $t \in[0, T]$ and $u \in \mathbb{R}^{N}$. So, putting together $\left(\mathscr{A}_{2}\right)$ and (26), one has

$$
\sqrt{m}\|u\|_{H_{T}^{1}} \leq\|u\| \leq \sqrt{M}\|u\|_{H_{T}^{1}}
$$

where $m=\min \{1, \nu\}$ and $M=\max \left\{1, \sum_{i, j=1}^{N}\left\|a_{i, j}\right\|_{\infty}\right\}$; that is, the norm $\|\cdot\|$ is equivalent to (23). At this point, since $\left(H_{T}^{1},\|\cdot\|_{H_{T}^{1}}\right)$ is compactly embedded in $C(0, T)$ (see [35]), due to (27) we claim that there exists a positive number $k$ such that

$$
\|u\|_{C} \leq k\|u\|
$$

where $k=(1 / \sqrt{m}) \max \{\sqrt{T}, 1 / \sqrt{T}\}$ and $\|u\|_{C}=\max _{t \in[0, T]}$ $|u(t)|$.

If $u \in H_{T}^{1}$, then $u$ is absolutely continuous and $\dot{u} \epsilon$ $L^{2}\left(0, T ; \mathbb{R}^{N}\right)$. In this case, $\Delta \dot{u}(t)=\dot{u}\left(t^{+}\right)-\dot{u}\left(t^{-}\right)=0$ is not necessarily valid for every $t \in(0, T)$ and the derivative $\dot{u}$ may present some discontinuities. This leads to the impulsive effects.

Following the ideas of [15], take $v \in H_{T}^{1}$ and multiply the two sides of the equality

$$
-\ddot{u}+A(t) u-\lambda \nabla F(t, u)-\mu \nabla G(t, u)=0
$$

by $v$ and integrate from 0 to $T$; we have

$$
\int_{0}^{T}[-\ddot{u}+A(t) u-\lambda \nabla F(t, u)-\mu \nabla G(t, u)] v d t=0 .
$$

Moreover, combining $\dot{u}(0)-\dot{u}(T)=0$, one has

$$
\begin{aligned}
& \int_{0}^{T}(\ddot{u}(t), v(t)) d t= \sum_{j=0}^{l} \int_{t_{j}}^{t_{j+1}}(\ddot{u}(t), v(t)) d t \\
&= \sum_{j=0}^{l}\left(\left(\dot{u}\left(t_{j+1}^{-}\right), v\left(t_{j+1}^{-}\right)\right)\right. \\
&\left.-\left(\dot{u}\left(t_{j}^{+}\right), v\left(t_{j}^{+}\right)\right)\right) \\
&-\sum_{j=0}^{l} \int_{t_{j}}^{t_{j+1}}(\dot{u}(t), \dot{v}(t)) d t \\
&= \sum_{j=0}^{l} \sum_{i=1}^{N}\left(\dot{u}^{i}\left(t_{j+1}^{-}\right) v^{i}\left(t_{j+1}^{-}\right)\right. \\
&-\int_{0}^{T}(\dot{u}(t), \dot{v}(t)) d t \\
&=-\sum_{j=1}^{l} \sum_{i=1}^{N} I_{i j}\left(\dot{u}^{i}\left(t_{j}\right)\right) v^{i}\left(t_{j}\right) \\
&\left.-\sum_{j=0}^{l} \int_{t_{j}}^{l} \sum_{i=1}^{t_{j+1}}\left(t_{j}^{+}\right) v^{i}\left(t_{j}^{+}\right)\right) \\
&= \dot{u}\left(t_{j}(t) v^{i}\left(t_{j}\right)\right. \\
&v(T)-\dot{u}(t)) d t
\end{aligned}
$$

Combining (30), we get

$$
\begin{aligned}
& \int_{0}^{T}(\dot{u}(t), \dot{v}(t))+(A(t) u(t), v(t)) d t \\
& \quad+\sum_{j=1}^{l} \sum_{i=1}^{N} I_{i j}\left(u^{i}\left(t_{j}\right)\right) v^{i}\left(t_{j}\right) \\
& \quad-\lambda \int_{0}^{T} \nabla F(t, u) v d t-\mu \int_{0}^{T} \nabla G(t, u) v d t=0 .
\end{aligned}
$$

Considering the above equality, we introduce the following concept of a weak solution to problem (9). 
Definition 13. One says that a function $u \in H_{T}^{1}$ is a weak solution to problem (9) if

$$
\begin{aligned}
& \int_{0}^{T}(\dot{u}(t), \dot{v}(t))+(A(t) u(t), v(t)) d t \\
& \quad+\sum_{j=1}^{l} \sum_{i=1}^{N} I_{i j}\left(u^{i}\left(t_{j}\right)\right) v^{i}\left(t_{j}\right) \\
& =\lambda \int_{0}^{T} \nabla F(t, u) v d t+\mu \int_{0}^{T} \nabla G(t, u) v d t
\end{aligned}
$$

holds for any $v \in H_{T}^{1}$.

In order to study problem (9), we will use the functionals $\Phi, J, \Psi: H_{T}^{1} \rightarrow \mathbb{R}$ defined by putting

$$
\begin{gathered}
\Phi(u)=\frac{1}{2}\|u\|^{2}+\sum_{j=1}^{l} \sum_{i=1}^{N} \int_{0}^{u^{i}\left(t_{j}\right)} I_{i j}(t) d t \\
=\phi(u)+\psi(u), \\
J(u):=\int_{0}^{T} F(t, u) d t, \quad \Psi(u):=\int_{0}^{T} G(t, u) d t,
\end{gathered}
$$

respectively, for every $u \in H_{T}^{1}$; here,

$$
\phi(u)=\frac{1}{2}\|u\|^{2}, \quad \psi(u)=\sum_{j=1}^{l} \sum_{i=1}^{N} \int_{0}^{u^{i}\left(t_{j}\right)} I_{i j}(t) d t .
$$

By the continuity of $I_{i j}, i=1,2, \ldots, N, j=1,2, \ldots, l$, we get that functional $\Phi$ is a continuous Gâteaux differential functional whose Gâteaux derivative is the functional $\Phi^{\prime}(u)$, given by

$$
\begin{aligned}
\left\langle\Phi^{\prime}(u), v\right\rangle= & \int_{0}^{T}(A(t) u(t), v(t))+(\dot{u}(t), \dot{v}(t)) d t \\
& +\sum_{j=1}^{l} \sum_{i=1}^{N} I_{i j}\left(u^{i}\left(t_{j}\right)\right) v^{i}\left(t_{j}\right),
\end{aligned}
$$

for any $u, v \in H_{T}^{1}$. On the other hand, condition (10) implies that $J$ and $\Psi$ are well defined, continuously Gâteaux differentiable in $H_{T}^{1}$. More precisely, their Gâteaux derivatives are

$$
\begin{aligned}
& \left\langle J^{\prime}(u), v\right\rangle=\int_{0}^{T} \nabla F(t, u) v d t, \\
& \left\langle\Psi^{\prime}(u), v\right\rangle=\int_{0}^{T} \nabla G(t, u) v d t,
\end{aligned}
$$

respectively, for every $u, v \in H_{T}^{1}$. Put

$$
\begin{gathered}
q=\sum_{i, j=1}^{N}\left\|a_{i, j}\right\|_{\infty}, \\
\lambda_{1} \inf \left\{\frac{\|u\|^{2}+2 \sum_{j=1}^{l} \sum_{i=1}^{N} \int_{0}^{u^{i}\left(t_{j}\right)} I_{i j}(t) d t}{2 \int_{0}^{T} F(t, u(t)) d t}: u \in H_{T}^{1},\right. \\
\left.\int_{0}^{T} F(t, u(t)) d t>0\right\},
\end{gathered}
$$

$\lambda_{2}$

$$
\begin{gathered}
=\left(\operatorname { m a x } \left\{0, \limsup _{\|u\| \rightarrow+\infty} \frac{2 \int_{0}^{T} F(t, u(t)) d t}{\|u\|^{2}+2 \sum_{j=1}^{l} \sum_{i=1}^{N} \int_{0}^{u^{i}\left(t_{j}\right)} I_{i j}(t) d t},\right.\right. \\
\left.\left.\quad \limsup _{|u| \rightarrow 0} \frac{2 \int_{0}^{T} F(t, u(t)) d t}{\|u\|^{2}+2 \sum_{j=1}^{l} \sum_{i=1}^{N} \int_{0}^{u^{i}\left(t_{j}\right)} I_{i j}(t) d t}\right\}\right) \\
D= \\
+\frac{\left(T-t_{l}\right)^{2}}{t_{1} t_{l}^{2}}+\frac{t_{1}}{3 t_{l}^{2}}\left(t_{l}^{2}+t_{l} T+T^{2}\right)+\left(t_{l}-t_{1}\right) \\
+\frac{T-t_{l}}{t_{l}^{2}}+\frac{1}{3 t_{l}^{2}}\left(T^{3}-t_{l}^{3}\right)>0 .
\end{gathered}
$$

Then we have the following.

Theorem 14 (see [16, Theorem 3.1]). Suppose that $\left(\mathscr{A}_{1}\right)-\left(\mathscr{A}_{2}\right)$ and $\left(\mathscr{I}_{1}\right)$ hold. Moreover, there exist a constant $p>0$ and constant vector $\bar{\xi}=\left(\bar{\xi}^{1}, \ldots, \bar{\xi}^{N}\right) \in \mathbb{R}^{N} \backslash\{0\}$ such that

$$
\begin{aligned}
& \left(\mathscr{R}_{1}\right) \max \left\{\lim \sup _{u \rightarrow 0}\left(\max _{t \in[0, T]} F(t, u) /|u|^{2}\right)\right. \text {, } \\
& \left.\lim \sup _{|u| \rightarrow+\infty}\left(\max _{t \in[0, T]} F(t, u) /|u|^{2}\right)\right\}<p ; \\
& \left(\mathscr{R}_{2}\right) p T k^{2}<\left(\int_{0}^{T} F(t, \bar{\xi}) d t /\left(q T|\bar{\xi}|^{2}+2 \sum_{j=1}^{l} \sum_{i=1}^{N} \int_{0}^{\bar{\xi}^{i}} I_{i j}(t)\right.\right.
\end{aligned}
$$

Then, for each compact interval $[a, b] \subset\left(\lambda_{1}, \lambda_{2}\right)$, there exists $R>0$ with the following property: for every $\lambda \in[a, b]$ and $G \in \mathscr{C}$ there exists $\delta>0$ such that, for each $\mu \in[0, \delta]$, problem (9) has at least three weak solutions whose norms are less than $R$.

Theorem 15 (see [16, Theorem 3.2]). Suppose that $\left(\mathscr{A}_{1}\right)-\left(\mathscr{A}_{2}\right)$ and $\left(\mathscr{I}_{1}\right)$ hold and there exist $h, q \in L^{1}\left(0, T ; \mathbb{R}^{+}\right)$, three positive constants $c, d, \alpha$ with $c>k d \sqrt{D m / 2}$, and $\alpha \in[0,2)$ such that

$$
\begin{aligned}
& \left(\mathscr{H}_{1}\right) \max _{t \in[0, T],|u| \leq c} F(t, u)<\left(D m d^{2} / T\right)\left(\int_{t_{1}}^{t_{l}} F(t, d \mathscr{E}) d t /\right. \\
& \left.\left(2 D M d^{2}+4 \sum_{j=1}^{l} \int_{0}^{d} I_{1 j}(t) d t\right)\right) ; \text { here, } \mathscr{E}=(1,0, \ldots, \\
& 0) \in \mathbb{R}^{N} ;
\end{aligned}
$$

$\left(\mathscr{H}_{2}\right) F(t, u) \geq 0$ for each $t \in\left[0, t_{1}\right] \cup\left[t_{l}, T\right],|u| \leq d T / t_{l}$; 
$\left(\mathscr{H}_{3}\right)|F(t, u)| \leq h(t)|u|^{\alpha}+q(t)$, for all $u \in \mathbb{R}^{N}$ and a.e. $t \in[0, T]$.

Then, there exist a nonempty open set $A \subset[0,+\infty)$ and $a$ positive number $R$ with the following property: for every $\lambda \in A$ and every $G \in \mathscr{C}$ there exists $\delta>0$ such that, for each $\mu \in[0, \delta]$, problem (9) has at least three weak solutions whose norms are less than $R$.

As a special case of $F(t, u)$, let $F(t, u)=b(t) f(u)$, where $b(t) \in L^{1}(0, T ; \mathbb{R}) \backslash\{0\}, f(u) \in C^{1}\left(\mathbb{R}^{N} ; \mathbb{R}\right)$. Then we get the following two corollaries.

Corollary 16 (see [16, Corollary 3.1]). Suppose that $\left(\mathscr{A}_{1}\right)$ $\left(\mathscr{A}_{2}\right)$ and $\left(\mathscr{I}_{1}\right)$ hold. Moreover, there exist a constant $p>0$ and constant vector $\bar{\xi}=\left(\bar{\xi}^{1}, \ldots, \bar{\xi}^{N}\right) \in \mathbb{R}^{N} \backslash\{0\}$ such that

$$
\begin{aligned}
& \left(\mathscr{R}_{1}\right)^{\prime} \max _{t \in[0, T]} b(t) \cdot \max \left\{\lim \sup _{u \rightarrow 0}\left(f(u) /|u|^{2}\right),\right. \\
& \left.\quad \limsup _{|u| \rightarrow+\infty}\left(f(u) /|u|^{2}\right)\right\}<p ; \\
& \left(\mathscr{R}_{2}\right)^{\prime} \\
& \begin{array}{l}
p T k^{2}<\left(f(\bar{\xi}) \int_{0}^{T} b(t) d t /\left(q T|\bar{\xi}|^{2}+2 \sum_{j=1}^{l} \sum_{i=1}^{N} \int_{0}^{\bar{\xi}^{i}} I_{i j}(t)\right.\right. \\
d t)) .
\end{array}
\end{aligned}
$$

Then, for each compact interval $[a, b] \subset\left(\lambda_{1}, \lambda_{2}\right)$, there exists $R>0$ with the following property: for every $\lambda \in[a, b]$ and $G \in \mathscr{C}$ there exists $\delta>0$ such that, for each $\mu \in[0, \delta]$, problem (9) has at least three weak solutions whose norms are less than R.

Corollary 17 (see [16, Corollary 3.2]). Suppose that $\left(\mathscr{A}_{1}\right)$ $\left(\mathscr{A}_{2}\right)$ and $\left(\mathscr{I}_{1}\right)$ hold. Furthermore, there exist five positive constants $c, d, \alpha, \beta$, and $\gamma$ with $c>k d \sqrt{D m / 2}$ and $\alpha \in[0,2)$ such that

$$
\begin{gathered}
\left(\mathscr{H}_{1}\right)^{\prime} \max _{|u| \leq c} f(u)<\left(D m d^{2} /\|b\|_{L^{1}}\right)\left(f(d \mathscr{E}) \int_{t_{1}}^{t_{l}} b(t) d t /\right. \\
\left.\left(D M d^{2}+2 \sum_{j=1}^{l} \int_{0}^{d} I_{1 j}(t) d t\right)\right)
\end{gathered}
$$

$\left(\mathscr{H}_{2}\right)^{\prime} b(t) f(u) \geq 0$ for any $t \in\left[0, t_{1}\right] \cup\left[t_{l}, T\right],|u| \leq d T / t_{l} ;$ $\left(\mathscr{H}_{3}\right)^{\prime}|f(u)| \leq \gamma+\beta|u|^{\alpha}$ for every $u \in \mathbb{R}^{N}$.

Then, there exist a nonempty open set $A \subset[0,+\infty)$ and $a$ positive number $R$ with the following property: for every $\lambda \in A$ and every $G \in \mathscr{C}$ there exists $\delta>0$ such that, for each $\mu \in[0, \delta]$, problem (9) has at least three weak solutions whose norms are less than $R$.

Finally, we consider the existence of infinitely many solutions for a class of impulsive systems with periodic boundary conditions

$$
\begin{gathered}
-\ddot{u}+A(t) u=\nabla W(t, u), \quad \text { a.e. } t \in[0, T], \\
\Delta\left(\dot{u}^{i}\left(t_{j}\right)\right)=\dot{u}^{i}\left(t_{j}^{+}\right)-\dot{u}^{i}\left(t_{j}^{-}\right)=I_{i j}\left(u^{i}\left(t_{j}\right)\right), \\
i=1,2, \ldots, N, \quad j=1,2, \ldots, l, \\
u(0)-u(T)=\dot{u}(0)-\dot{u}(T)=0,
\end{gathered}
$$

where $A:[0, T] \rightarrow \mathbb{R}^{N \times N}$ is a continuous map from the interval $[0, T]$ to the set of $N$-order symmetric matrices, $T$ is a real positive number, $u(t)=\left(u^{1}(t), u^{2}(t), \ldots, u^{N}(t)\right), t_{j}$, $j=1,2, \ldots, l$, are the instants where the impulses occur and $0=t_{0}<t_{1}<t_{2}<\cdots<t_{l}<t_{l+1}=T, I_{i j}: \mathbb{R} \rightarrow \mathbb{R}(i=$ $1,2, \ldots, N, j=1,2, \ldots, l)$ are continuous, and $W:[0, T] \times$ $\mathbb{R}^{N} \rightarrow \mathbb{R}$ are measurable with respect to $t$, for every $u \in \mathbb{R}^{N}$, continuously differentiable in $u$, for almost every $t \in[0, T]$, and satisfy the following standard summability condition:

$$
\sup _{|u| \leq b} \max \{|W(\cdot, u)|,|\nabla W(\cdot, u)|\} \in L^{1}(0, T), \quad \forall b>0 .
$$

Now we state our main results.

Theorem 18 (see [36, Theorem 1.1]). Assume that $W$ is even in $u$ and the following conditions hold:

$$
\begin{aligned}
& \left(\mathscr{H}_{1}\right) I_{i j}(i=1, \ldots, N, j=1,2, \ldots, l) \text { are odd and satisfy } \\
& 2 \int_{0}^{s} I_{i j}(t) d t-I_{i j}(s) s \geq 0, \quad \int_{0}^{s} I_{i j}(t) d t \geq 0 \quad \forall s \in \mathbb{R} ;
\end{aligned}
$$

$\left(\mathscr{H}_{2}\right)$ there exist constants $a_{i j}, b_{i j}>0$ and $\gamma_{i j} \in[0,1)$ such that

$$
\left|I_{i j}(s)\right| \leq a_{i j}+b_{i j}|s|^{\gamma_{i j}} \quad \text { for } s \in \mathbb{R}
$$

$\left(\mathscr{H}_{3}\right) \lim _{|u| \rightarrow+\infty}\left(W(t, u) / u^{2}\right)=+\infty$ uniformly for $t \in$ $[0, T]$;

$\left(\mathscr{H}_{4}\right) W(t, 0) \equiv 0,0 \leq W(t, u)=o\left(|u|^{2}\right)$ as $|u| \rightarrow 0$ uniformly for $t \in[0, T]$;

$\left(\mathscr{H}_{5}\right)$ there exist constants $\alpha>1,1<\beta<1+((\alpha-1) / \alpha)$, $c_{1}, c_{2}>0$, and $L>0$ such that for every $t \in[0, T]$ and $u \in \mathbb{R}^{N}$ with $|u| \geq L$,

$$
(\nabla W(t, u), u)-2 W(t, u) \geq c_{1}|u|^{\alpha},|\nabla W(t, u)| \leq c_{2}|u|^{\beta} .
$$

Then problem (39) has infinitely many weak solutions.

Theorem 19 (see [36, Theorem 1.2]). Assume that the following conditions hold:

$\left(\mathcal{S}_{1}\right) I_{i j}(i=1,2, \ldots, N, j=1,2, \ldots, l)$ are odd and satisfy $I_{i j}(s) s \geq 0$ for any $s \in \mathbb{R}$;

$\left(\mathcal{S}_{2}\right)$ there exist constants $b_{i j}>0$ and $\gamma_{i j} \in[0,1)$ such that

$$
\left|I_{i j}(s)\right| \leq b_{i j}|s|^{\gamma_{i j}} \quad \text { for } s \in \mathbb{R}
$$

$\left(\mathcal{S}_{3}\right) W(t, u)=(\rho / 2)|u|^{2}+F(t, u)$, where $\rho$ is a positive constant and not a spectrum point of $-\left(d^{2} / d t^{2}\right)+A, F$ is even in $u$, and $F(t, 0) \equiv 0$;

$\left(\delta_{4}\right)$ there exist $\delta_{1}, \delta_{2} \in[1,2)$ with $\delta_{1}<$ $\min _{1 \leq i \leq N, 1 \leq j \leq l}\left\{\gamma_{i j}\right\}+1$, and $d_{1}, d_{2}>0$ such that

$$
\begin{array}{r}
d_{1}|u|^{\delta_{1}} \leq F(t, u),|\nabla F(t, u)| \leq d_{2}|u|^{\delta_{2}-1}, \\
\forall(t, u) \in[0, T] \times \mathbb{R}^{N} .
\end{array}
$$

Then problem (39) has infinitely many weak solutions. 
Theorem 20 (see [36, Theorem 1.3]). Assume that $W$ is even in $u,\left(\mathcal{S}_{1}\right)-\left(\mathcal{S}_{2}\right)$ and the following conditions hold:

$$
\begin{aligned}
& \left(\mathscr{J}_{1}\right) W(t, 0) \equiv 0 \text { for any } t \in[0, T] \text {; } \\
& \left(\mathscr{J}_{2}\right) \text { there are constants } k_{1}>0 \text { and } \zeta_{1} \in[1,2) \text { with } \zeta_{1}< \\
& \min _{1 \leq i \leq N, 1 \leq j \leq l}\left\{\gamma_{i j}\right\}+1 \text { such that } \\
& W(t, u) \geq k_{1}|u|^{\zeta_{1}}, \quad \text { for any }(t, u) \in[0, T] \times \mathbb{R}^{N} ; \\
& |\nabla W(t, u)| \leq k_{2}|u|^{\zeta_{2}-1}, \quad \text { for any }(t, u) \in[0, T] \times \mathbb{R}^{N} .
\end{aligned}
$$

Then problem (39) has infinitely many weak solutions.

\section{Periodic Solutions}

Firstly, we consider periodic solutions for second-order regular impulsive problems with a perturbation:

$$
\begin{gathered}
u^{\prime \prime}(t)-V_{u}(t, u)=f(t), \quad \text { for } t \in\left(s_{k-1}, s_{k}\right), \\
\Delta u^{\prime}\left(s_{k}\right)=g_{k}\left(u\left(s_{k}\right)\right),
\end{gathered}
$$

where $k \in \mathbb{Z}, u \in \mathbb{R}^{n}, \Delta u^{\prime}\left(s_{k}\right)=u^{\prime}\left(s_{k}^{+}\right)-u^{\prime}\left(s_{k}^{-}\right)$with $u^{\prime}\left(s_{k}^{ \pm}\right)=$ $\lim t \rightarrow s_{k}^{ \pm}, V_{u}(t, u)=\operatorname{grad}_{u} V(t, u), f \in C\left(\mathbb{R}, \mathbb{R}^{n}\right), g_{k}(u)=$ $\operatorname{grad}_{u} G_{k}(u), G_{k} \in C^{1}\left(\mathbb{R}^{n}, \mathbb{R}^{n}\right)$ for each $k \in \mathbb{Z}$, and there exist $m \in \mathbb{N}$ and $T \in \mathbb{R}^{+}$such that $0=s_{0}<s_{1}<\cdots<s_{m}=T$, $s_{k+m}=s_{k+T}$, and $g_{k+m} \equiv g_{k}$ for all $k \in \mathbb{Z}$ (i.e., $g_{k}$ is $m$-periodic in $k$ ).

Theorem 21 (see [20, Theorem 1]). Assume that $f$ is continuous and T-periodic and $g_{k}$ is continuous, $m$-periodic in $k$. Furthermore, assume that $V$ and $g_{k}$ satisfy the following conditions:

$\left(V_{1}\right) V: \mathbb{R} \times \mathbb{R}^{n} \rightarrow \mathbb{R}$ is continuous differentiable and $T$ periodic, and there exist positive constants $b_{1}, b_{2}>0$ such that

$$
b_{1}|u|^{2} \leq-V(t, u) \leq|u|^{2}, \quad \forall(t, u) \in[0, T] \times \mathbb{R}^{n}
$$

$\left(V_{2}\right)-V(t, u) \leq-V_{u}(t, u) u \leq-2 V(t, u)$ for all $(t, u) \in$ $[0, T] \times \mathbb{R}^{n} ;$

$\left(g_{1}\right)$ there exists $\mu>2$ such that

$g_{k}(u) u \leq \mu G_{k}(u)<0, \quad$ for $u \in \mathbb{R}^{n} \backslash\{0\}, k=1,2, \ldots, m$.

Then there exists $\bar{b}_{f}>0$ such that, for any positive integer $l$, if $b_{f, 1} \leq \bar{b}_{f} l^{-1 / 2}$, then problem (48) possesses at least one nonzero $l T$-periodic solution.

Theorem 22 (see [20, Theorem 1]). Suppose that $V$ is even about $u$ and satisfies $\left(V_{1}\right)-\left(V_{2}\right), f \equiv 0$, and $g_{k}$ is continuous, odd about $u, m$-periodic in $k$, and satisfies the following: $\left(g_{2}\right)$ for all $k=1,2, \ldots, m$,

$$
\liminf _{|u| \rightarrow \infty} \frac{G_{k}(u)}{|u|^{2}} \geq 0 .
$$

Then there exists $C_{g}>0$ such that if

$$
\limsup _{|u| \rightarrow 0} \frac{G_{k}(u)}{|u|^{2}} \leq-C_{g},
$$

then problem (48) possesses at least Imn distinct pairs of $l T$-periodic solutions generated by impulses.

Theorem 23 (see [14, Theorem 1]). Assume that $f \equiv 0$ and $g_{k}$ is continuous, $m$-periodic in $k$ satisfying $\left(g_{1}\right)$. Furthermore, assume that $V$ satisfies the following condition:

$\left(V_{3}\right) V: \mathbb{R} \times \mathbb{R}^{n} \rightarrow \mathbb{R}$ is continuous differentiable and $T$ periodic, and $V(t, u) \geq(1 / 2) V_{u}(t, u) u>0$ for all $t \in$ $[0, T]$ and $u \in \mathbb{R}^{n} \backslash\{0\}$.

Then problem (48) possesses one nonzero periodic solution generated by impulses.

Corollary 24 (see [14, Corollary 1]). Assume that $f \equiv 0$ and $g_{k}$ is continuous, $m$-periodic in $k$ satisfying $\left(g_{1}\right)$. Furthermore, assume that $V$ satisfies the following condition:

$\left(V_{4}\right) V_{u}(t, u)=\alpha u+W_{u}(t, u)$ for all $t \in[0, T]$ and $u \in$ $\mathbb{R}^{n}$, where $\alpha>2 / \mu$ for some $\mu>2$ and $W(t, u) \geq$ $(1 / 2) W_{u}(t, u) u>0$ for all $t \in[0, T]$ and $u \in \mathbb{R}^{n} \backslash\{0\}$.

Then problem (48) possesses one nonzero periodic solution generated by impulses.

Theorem 25 (see [14, Theorem 2]). Assume that $f \equiv 0$ and $V$ and $g_{k}$ satisfy the following conditions:

$\left(V_{5}\right) V(t, u) \geq(1 / 2) V_{u}(t, u) u \geq 0$ for all $(t, u) \in[0, T] \times \mathbb{R}^{n}$;

$\left(g_{3}\right) g_{k}(u)=2 u+w_{k}(u)$, where $w_{k}=\operatorname{grad}_{u} W_{k}(u)$ and satisfies the fact that there exists $\mu>2$ such that

$$
\begin{array}{r}
w_{k}(u) u \leq \mu W_{k}(u)<0, \quad \text { for } u \in \mathbb{R}^{n} \backslash\{0\}, \\
k=1,2, \ldots, m .
\end{array}
$$

Then problem (48) possesses one nonzero periodic solution generated by impulses.

Now we discuss $T$-periodic solution to second-order impulsive problems with singularity as follows:

$$
\begin{gathered}
u^{\prime \prime}(t)-\frac{1}{u^{\alpha}(t)}=e(t), \quad \text { a.e. } t \in(0, T), \\
\Delta u^{\prime}\left(t_{j}\right)=I_{j}\left(u\left(t_{j}\right)\right), \quad j=1,2, \ldots, p-1,
\end{gathered}
$$

where $\alpha \geq 1$, e $\in L^{1}([0, T], \mathbb{R})$ is $T$-periodic, $\Delta u^{\prime}\left(t_{j}\right)=$ $u^{\prime}\left(t_{j}^{+}\right)-u^{\prime}\left(t_{j}^{-}\right)$with $u^{\prime}\left(t_{j}^{ \pm}\right)=\lim _{t \rightarrow t_{j}^{ \pm}} u^{\prime}(t) ; 0=t_{0}<t_{1}<$ $t_{2}<\cdots<t_{p-1}<t_{p}=T$ and $t_{j+p}=t_{j}+T ; I_{j} \in C(\mathbb{R}, \mathbb{R})(j=$ $1,2, \ldots, p-1)$ and $I_{j+p} \equiv I_{j}$. 
Theorem 26 (see [18, Theorem 1.2]). Assume that

$\left(\mathcal{S}_{1}\right) e \in L^{1}([0, T], \mathbb{R})$ is $T$-periodic and $\int_{0}^{T} e(t) d t \geq 0$;

$\left(\mathcal{S}_{2}\right)$ there exist two constants $m, M$ such that, for any $s \in \mathbb{R}$,

$$
m \leq I_{j}(s) \leq M, \quad j=1,2, \ldots, p-1,
$$

where

$$
m \leq M<-\frac{1}{p-1} \int_{0}^{T} e(t) d t \leq 0 .
$$

Then problem (54) has at least a positive T-periodic solution.

Set

$$
\begin{aligned}
& H_{T}^{1}=\{u: \mathbb{R} \longrightarrow \mathbb{R} \mid u \text { is absolutely continuous, } \\
& \left.\qquad u^{\prime} \in L^{2}((0, T), \mathbb{R}), \text { and } u(t)=u(t+T) \text { for } t \in \mathbb{R}\right\}
\end{aligned}
$$

with the inner product

$$
(u, v)=\int_{0}^{T} u(t) v(t) d t+\int_{0}^{T} u^{\prime}(t) v^{\prime}(t) d t, \quad \forall u, v \in H_{T}^{1}
$$

The corresponding norm is defined by

$$
\|u\|_{H_{T}^{1}}=\left(\int_{0}^{T}|u(t)|^{2} d t+\int_{0}^{T}\left|u^{\prime}(t)\right|^{2} d t\right)^{1 / 2}, \quad \forall u \in H_{T}^{1} .
$$

Then $H_{T}^{1}$ is a Banach space (in fact it is a Hilbert space).

In order to study problem (54), for any $\lambda \in(0,1)$, we consider the following modified problem:

$$
\begin{gathered}
u^{\prime \prime}(t)+f_{\lambda}(u(t))=e(t), \quad \text { a.e. } t \in(0, T), \\
\Delta u^{\prime}\left(t_{j}\right)=I_{j}\left(u\left(t_{j}\right)\right), \quad j=1,2, \ldots, p-1 .
\end{gathered}
$$

Following the ideas of [15], we introduce the following concept of a weak solution to problem (60).

Definition 27. One says that a function $u \in H_{T}^{1}$ is a weak solution to problem (60) if

$$
\begin{aligned}
& \int_{0}^{T} u^{\prime}(t) v^{\prime}(t) d t+\sum_{j=1}^{p-1} I_{j}\left(u\left(t_{j}\right)\right) v\left(t_{j}\right) \\
& \quad-\int_{0}^{T} f_{\lambda}(u(t)) v(t) d t+\int_{0}^{T} e(t) v(t) d t=0
\end{aligned}
$$

holds for any $v \in H_{T}^{1}$.

Let $F_{\lambda} \in C^{1}(\mathbb{R}, \mathbb{R})$ be defined by

$$
F_{\lambda}(s)=\int_{1}^{s} f_{\lambda}(t) d t
$$

and consider the functional

$$
\Phi_{\lambda}: H_{T}^{1} \longrightarrow \mathbb{R}
$$

defined by

$$
\begin{aligned}
\Phi_{\lambda}(u):= & \frac{1}{2} \int_{0}^{T}\left|u^{\prime}(t)\right|^{2} d t+\sum_{j=1}^{p-1} \int_{0}^{u\left(t_{j}\right)} I_{j}(s) d s \\
& -\int_{0}^{T} F_{\lambda}(u(t)) d t+\int_{0}^{T} e(t) u(t) d t .
\end{aligned}
$$

Clearly, $\Phi_{\lambda}$ is well defined on $H_{T}^{1}$, continuously Gâteaux differentiable functional whose Gâteaux derivative is the functional $\Phi_{\lambda}^{\prime}(u)$, given by

$$
\begin{aligned}
\Phi_{\lambda}^{\prime}(u) v= & \int_{0}^{T} u^{\prime}(t) v^{\prime}(t) d t+\sum_{j=1}^{p-1} I_{j}\left(u\left(t_{j}\right)\right) v\left(t_{j}\right) \\
& -\int_{0}^{T} f_{\lambda}(u(t)) v(t) d t+\int_{0}^{T} e(t) v(t) d t,
\end{aligned}
$$

for any $v \in H_{T}^{1}$. Moreover, it is easy to verify that $\Phi_{\lambda}$ is weakly lower semicontinuous. Indeed, if $\left\{u_{n}\right\} \subset H_{T}^{1}, u \in H_{T}^{1}$, and $u_{n} \rightarrow u$, then $\left\{u_{n}\right\}$ converges uniformly to $u$ on $[0, T]$ and $u_{n} \rightarrow u$ on $L^{2}([0, T])$, and combining the fact that $\liminf _{n \rightarrow \infty}\left\|u_{n}\right\|_{H_{T}^{1}} \geq\left\|u_{n}\right\|_{H_{T}^{1}}$, we have

$$
\begin{aligned}
\liminf _{n \rightarrow \infty} \Phi_{\lambda}\left(u_{n}\right)=\liminf _{n \rightarrow \infty}( & \frac{1}{2}\left\|u_{n}\right\|_{H_{T}^{1}}^{2}-\frac{1}{2} \int_{0}^{T}\left|u_{n}(t)\right|^{2} d t \\
& +\sum_{j=1}^{p-1} \int_{0}^{u_{n}\left(t_{j}\right)} I_{j}(s) d s \\
& -\int_{0}^{T} F_{\lambda}\left(u_{n}(t)\right) d t \\
& \left.+\int_{0}^{T} e(t) u_{n}(t) d t\right) \\
\geq & \frac{1}{2} \int_{0}^{T}\left|u^{\prime}(t)\right|^{2} d t+\sum_{j=1}^{p-1} \int_{0}^{u\left(t_{j}\right)} I_{j}(s) d s \\
& -\int_{0}^{T} F_{\lambda}(u(t)) d t+\int_{0}^{T} e(t) u(t) d t \\
= & \Phi_{\lambda}(u) .
\end{aligned}
$$

By the standard discussion, the critical points of $\Phi_{\lambda}$ are the weak solutions of problem (1); see $[15,16]$.

The following version of the mountain pass theorem will be used in our argument.

Theorem 28 (see Theorem 4.10, [35]). Let $X$ be a Banach space and let $\varphi \in C^{1}(X, \mathbb{R})$. Assume that there exist $x_{0}, x_{1} \in X$ and a bounded open neighborhood $\Omega$ of $x_{0}$ such that $x_{1} \in X \backslash \bar{\Omega}$ and

$$
\max \left\{\varphi\left(x_{0}\right), \varphi\left(x_{1}\right)\right\}<\inf _{x \in \partial \Omega} \varphi(x) .
$$


Let

$$
\begin{gathered}
\Gamma=\left\{h \in C([0,1], X): h(0)=x_{0}, h(1)=x_{1}\right\}, \\
c=\inf _{h \in \Gamma s \in[0,1]} \max _{\varphi} \varphi(h(s)) .
\end{gathered}
$$

If $\varphi$ satisfies the (PS) condition, that is, a sequence $\left\{u_{n}\right\}$ in $X$ satisfying $\varphi\left(u_{n}\right)$ is bounded and $\varphi^{\prime}\left(u_{n}\right) \rightarrow 0$ as $n \rightarrow \infty$ has a convergent subsequence, then $c$ is a critical value of $\varphi$ and $c>\max \left\{\varphi\left(x_{0}\right), \varphi\left(x_{1}\right)\right\}$.

If $I_{j}\left(u\left(t_{j}\right)\right) \equiv b_{j}(j=1,2, \ldots, p-1)$, then we have the following result of problem (54).

Theorem 29 (see [17, Theorem 1.2]). Assume that $e \in$ $L^{1}([0, T], \mathbb{R})$ is $T$-periodic. Then problem (54) has a positive $T$-periodic weak solution $u \in H_{T}^{1}$ if and only if $\sum_{j=1}^{p-1} b_{j}+$ $\int_{0}^{T} e(t) d t<0$.

Remark 30. From Theorem 29 we can see that if $\int_{0}^{T} e(t) d t \geq 0$ but $\sum_{j=1}^{p-1} b_{j}+\int_{0}^{T} e(t) d t<0$, then problem (54) still admits a positive $T$-periodic solution. This shows that the existence of positive $T$-periodic solution to problem (54) depends on the forced term $e$ and impulsive functions $b_{j}$ together, not single one.

\section{Homoclinic Solutions}

In this section, we are interested in the existence of homoclinic solutions for problem (48).

First we consider the case of $f \equiv 0$. When $V_{u}(t, u)$ is asymptotically linear, we show that there exists a nonzero homoclinic solution as the limit of a sequence $2 l T$-periodic solutions as $l$ goes to $\infty$, and when $V_{u}(t, u)$ is sublinear, we attain a nonzero homoclinic solution in the same way by strengthening the effect of impulses. The results are as follows.

Theorem 31 (see [14, Theorem 3]). Supposing that $f \equiv 0, V$ is $T$-periodic in $t$ and satisfies condition $\left(V_{4}\right)$, and $g_{k}$ satisfies condition $\left(g_{1}\right)$, then problem (48) possesses at least one nonzero homoclinic solution generated by impulses.

Theorem 32 (see [14, Theorem 4]). Supposing that $f \equiv 0, V$ is $T$-periodic in $t$ and satisfies condition $\left(V_{5}\right)$, and $g_{k}$ satisfies condition $\left(g_{3}\right)$, then problem (48) possesses at least one nonzero homoclinic solution generated by impulses.

If $n=1$ and $g_{k}(u) \equiv I(u)$ for all $k=1,2, \ldots, m$, then we have the following result for problem (48) when $V_{u}$ satisfies the superlinear condition at infinity on $u$.

Theorem 33 (see [13, Theorem 1.1]). Assume that $f \equiv 0$ and the following conditions hold:

$\left(H_{1}\right)$ there exists a positive number $T$ such that

$$
\begin{array}{r}
V_{u}(t+T, u)=V_{u}(t, u), \quad V(t+T, u)=V(t, u) \\
\forall(t, u) \in \mathbb{R}^{2} ;
\end{array}
$$

$\left(H_{2}\right) \lim _{u \rightarrow 0} \frac{V_{u}(t, u)}{u}=0$ uniformly for $t \in \mathbb{R}$

$\left(H_{3}\right)$ there exists a $\mu>2$ such that

$u V_{u}(t, u) \geq \mu V(t, u)>0, \quad \forall(t, u) \in \mathbb{R} \times \mathbb{R} \backslash\{0\}$

$\left(H_{4}\right)$ there exist constants $a_{0}>0$ and $a_{1}>0$ such that

$$
\begin{aligned}
& V(t, u) \geq a_{0}|u|^{\mu}, \quad \forall|u| \geq 1, t \in \mathbb{R} ; \\
& V(t, u) \leq a_{0}|u|^{\mu}, \quad \forall|u| \leq 1, t \in \mathbb{R} ;
\end{aligned}
$$

$\left(H_{5}\right)$ there exists a constant $b$, with $0<b<((\mu-2) /(\mu+$ 2)Tm), such that

$$
|I(u)| \leq b|u|, \quad 2 \int_{0}^{u} I(t) d t-I(u) u \leq 0 .
$$

Then there exists a nontrivial weak homoclinic solution to problem (48).

The proof of Theorem 33 is based on the following version of Mountain pass theorem.

Theorem 34 (see [37]). Let $E$ be a Banach space and let $\varphi \in$ $C^{1}(E, \mathbb{R}), e \in E, r>0$ be such that $\|e\|>r$ and $b:=$ $\inf _{\|y\|=r} \varphi(y)>\varphi(0) \geq \varphi(e)$. Let

$$
\begin{gathered}
\Gamma=\left\{h \in C([0,1], X): h(0)=x_{0}, h(1)=x_{1}\right\}, \\
c:=\inf _{h \in \Gamma s \in[0,1]} \max _{s}(h(s)) .
\end{gathered}
$$

Then, for each $\epsilon>0, \delta>0$, there exists $y \in E$ such that

$$
\begin{aligned}
& \left(M_{1}\right) c-2 \epsilon \leq \varphi(y) \leq c+2 \epsilon \\
& \left(M_{2}\right) \operatorname{dist}(y, E) \leq 2 \delta \\
& \left(M_{3}\right)\left\|\varphi^{\prime}(y)\right\| \leq 8 \epsilon / \delta .
\end{aligned}
$$

Next we consider the case of $f \not \equiv 0$ for problem (48).

Theorem 35 (see [20, Theorem 3]). Assume that $V$ satisfies conditions $\left(V_{1}\right)-\left(V_{2}\right)$ and $g_{k}$ satisfies $\left(g_{1}\right)$. Furthermore, $g_{k}$ satisfies the following condition:

$\left(g_{4}\right) g_{k} \in C(\mathbb{R})$ is m-periodic in $k$, and $\lim _{|u| \rightarrow 0}\left(g_{k}(u) /\right.$ $|u|)=0$ for $k=1,2, \ldots, m$.

Then there exists $\bar{b}_{f}>0$ such that if $\int_{\mathbb{R}}|f(t)|^{2} d t \leq \bar{b}_{f}^{2}$, then problem (48) possesses at least one nonzero homoclinic solution.

If $V$ satisfies some other conditions, then we still obtain the existence result for problem (48).

Theorem 36 (see [19, Theorem 1]). Assume that the following conditions hold:

$\left(V_{6}\right) V(t, u)=-K(t, u)+W(t, u)$, where $K, W \in C^{1}(\mathbb{R} \times$ $\left.\mathbb{R}^{n}, \mathbb{R}\right)$, and is $T$-periodic in its first variable; 
$\left(K_{1}\right)$ there exist constants $a_{1}>0$ and $\gamma \in(1,2]$ such that for all $(t, u) \in[0, T] \times \mathbb{R}^{n}$

$$
K(t, 0) \equiv 0, \quad K(t, u) \geq a_{1}|u|^{\gamma}
$$

$\left(K_{2}\right)$ there exists $\theta \in(1,2]$ such that

$K(t, u) \leq K_{u}(t, u) u \leq \theta K(t, u), \quad \forall(t, u) \in[0, T] \times \mathbb{R}^{n}$

$\left(W_{1}\right) W(t, 0)=0, W_{u}(t, u)=o(|u|)$, as $|u| \rightarrow 0$ uniformly for $t \in[0, T]$;

$\left(W_{2}\right)$ there exists $r>2$ and $b_{1}>0$ such that

$$
W(t, u) \leq b_{1}|u|^{r}, \quad \forall(t, u) \in[0, T] \times \mathbb{R}^{n}
$$

$\left(W_{3}\right)$ there exist $\mu>1, a_{2}>0$, and $\beta(t) \in L^{1}\left(\mathbb{R}, \mathbb{R}^{+}\right)$such that $\mu>r-\gamma$ and

$$
\begin{array}{r}
W_{u}(t, u) u-2 W(t, u) \geq a_{2}|u|^{\mu}-\beta(t), \\
\forall(t, u) \in[0, T] \times \mathbb{R}^{n} ;
\end{array}
$$

$\left(W_{4}\right) W(t, u) /|u|^{2} \rightarrow+\infty$ as $|u| \rightarrow+\infty$ uniformly in $t \epsilon$ $[0, T]$

$\left(G_{1}\right) G_{k}(0)=0$ and $\lim _{|u| \rightarrow 0} \frac{g_{k}(u)}{|u|}=0$ for $k=1,2, \ldots, m$;

$\left(G_{2}\right)$ there exists $b_{2}>0$ such that $G_{k}(u) \leq b_{2}|u|^{r}, u \in \mathbb{R}^{n}$, $k=1,2, \ldots, m$;

$\left(G_{3}\right) g_{k}(u) u-2 G_{k}(u) \geq 0, u \in \mathbb{R}^{n} \backslash\{0\}, k=1,2, \ldots, m ;$

$\left(F_{1}\right) f \in C\left(\mathbb{R}, \mathbb{R}^{n}\right) \cap L^{2}\left(\mathbb{R}, \mathbb{R}^{n}\right) \cap L^{\mu /(\mu-1)}$ satisfying

$$
\left(\int_{\mathbb{R}}|f(t)|^{2} d t\right)^{1 / 2}<\frac{1}{C} \min \left\{\frac{1}{2}, a_{1}-b_{1}-M\right\},
$$

where $M:=\sup \left\{G_{k}(u): k=1,2, \ldots, m,|u|=1\right\}$ and $a_{1}>$ $b_{1}+M$.

Then problem (48) possesses at least one nonzero homoclinic solution.

\section{Conflict of Interests}

The authors declare that there is no conflict of interests regarding the publication of this paper.

\section{Acknowledgments}

J. Sun was supported by the National Natural Science Foundation of China (Grant nos. 11201270 and 11271372), Shandong Natural Science Foundation (Grant no. ZR2012AQ010), and Young Teacher Support Program of Shandong University of Technology.

\section{References}

[1] T. E. Carter, "Optimal impulsive space trajectories based on linear equations," Journal of Optimization Theory and Applications, vol. 70, no. 2, pp. 277-297, 1991.

[2] T. E. Carter, "Necessary and sufficient conditions for optimal impulsive rendezvous with linear equations of motion," Dynamics and Control, vol. 10, no. 3, pp. 219-227, 2000.

[3] J. Chu and J. J. Nieto, "Impulsive periodic solutions of firstorder singular differential equations," Bulletin of the London Mathematical Society, vol. 40, no. 1, pp. 143-150, 2008.

[4] W. Li and H. Huo, "Global attractivity of positive periodic solutions for an impulsive delay periodic model of respiratory dynamics," Journal of Computational and Applied Mathematics, vol. 174, no. 2, pp. 227-238, 2005.

[5] C. Li, X. Liao, X. Yang, and T. Huang, "Impulsive stabilization and synchronization of a class of chaotic delay systems," Chaos, vol. 15, Article ID 043103, 9 pages, 2005.

[6] A. F. B. A. Prado, "Bi-impulsive control to build a satellite constellation," Nonlinear Dynamics and Systems Theory, vol. 5, no. 2, pp. 169-175, 2005.

[7] R. P. Agarwal, D. Franco, and D. O’Regan, "Singular boundary value problems for first and second order impulsive differential equations," Aequationes Mathematicae, vol. 69, no. 1-2, pp. 8396, 2005.

[8] B. Ahmad and J. J. Nieto, "Existence and approximation of solutions for a class of nonlinear impulsive functional differential equations with anti-periodic boundary conditions," Nonlinear Analysis: Theory, Methods \& Applications, vol. 69, no. 10, pp. 3291-3298, 2008.

[9] V. Lakshmikantham, D. D. Bainov, and P. S. Simeonov, Theory of Impulsive Differential Equations, vol. 6, World Scientific, Singapore, 1989.

[10] J. Li, J. J. Nieto, and J. Shen, "Impulsive periodic boundary value problems of first-order differential equations," Journal of Mathematical Analysis and Applications, vol. 325, no. 1, pp. 226236, 2007.

[11] J. J. Nieto, "Basic theory for nonresonance impulsive periodic problems of first order," Proceedings of the American Mathematical Society, vol. 125, pp. 2599-2604, 1997.

[12] A. M. Samoilenko and N. A. Perestyuk, Impulsive Differential Equations, vol. 14, World Scientific, Singapore, 1995.

[13] H. Fang and H. Duan, "Existence of nontrivial weak homoclinic orbits for second-order impulsive differential equations," Boundary Value Problems, vol. 2012, article 138, 13 pages, 2012.

[14] X. Han and H. Zhang, "Periodic and homoclinic solutions generated by impulses for asymptotically linear and sublinear Hamiltonian system," Journal of Computational and Applied Mathematics, vol. 235, no. 5, pp. 1531-1541, 2011.

[15] J. J. Nieto and D. O’Regan, "Variational approach to impulsive differential equations," Nonlinear Analysis: Real World Applications, vol. 10, no. 2, pp. 680-690, 2009.

[16] J. Sun, H. Chen, J. J. Nieto, and M. Otero-Novoa, "The multiplicity of solutions for perturbed second-order Hamiltonian systems with impulsive effects," Nonlinear Analysis: Theory, Methods \& Applications, vol. 72, no. 12, pp. 4575-4586, 2010.

[17] J. Sun and J. Chu, "A suffcient and necessary condition on T-periodic solution for a singular problem with impulses," Electronic Journal of Differential Equations, vol. 94, pp. 1-10, 2014. 
[18] J. Sun, J. Chu, and H. Chen, "Periodic solution generated by impulses for singular differential equations," Journal of Mathematical Analysis and Applications, vol. 404, no. 2, pp. 562-569, 2013.

[19] J. Xie, Z. Luo, and G. Chen, "Homoclinic solutions for a class of the second-order impulsive Hamiltonian systems," Abstract and Applied Analysis, vol. 2013, Article ID 583107, 8 pages, 2013.

[20] H. Zhang and Z. Li, "Periodic and homoclinic solutions generated by impulses," Nonlinear Analysis: Real World Applications, vol. 12, no. 1, pp. 39-51, 2011.

[21] Z. Zhang and R. Yuan, "An application of variational methods to Dirichlet boundary value problem with impulses," Nonlinear Analysis: Real World Applications, vol. 11, no. 1, pp. 155-162, 2010.

[22] J. Zhou and Y. Li, "Existence and multiplicity of solutions for some Dirichlet problems with impulsive effects," Nonlinear Analysis: Theory, Methods \& Applications, vol. 71, no. 7-8, pp. 2856-2865, 2009.

[23] R. P. Agarwal, T. G. Bhaskar, and K. Perera, "Some results for impulsive problems via Morse theory," Journal of Mathematical Analysis and Applications, vol. 409, no. 2, pp. 752-759, 2014.

[24] H. Chen and J. Sun, "An application of variational method to second-order impulsive differential equation on the half-line," Applied Mathematics and Computation, vol. 217, no. 5, pp. 18631869, 2010.

[25] J. Sun and H. Chen, "Variational method to the impulsive equation with Neumann boundary conditions," Boundary Value Problems, vol. 2009, Article ID 316812, 17 pages, 2009.

[26] J. Sun and H. Chen, "Multiplicity of solutions for a class of impulsive differential equations with Dirichlet boundary conditions via variant fountain theorems," Nonlinear Analysis: Real World Applications, vol. 11, no. 5, pp. 4062-4071, 2010.

[27] Y. Tian and W. Ge, "Applications of variational methods to boundary-value problem for impulsive differential equations," Proceedings of the Edinburgh Mathematical Society, vol. 51, no. 2, pp. 509-527, 2008.

[28] Y. Tian and W. Ge, "Variational methods to Sturm-Liouville boundary value problem for impulsive differential equations," Nonlinear Analysis: Theory, Methods \& Applications, vol. 72, no. 1, pp. 277-287, 2010.

[29] Y. Tian and W. Ge, "Multiple solutions of impulsive SturmLiouville boundary value problem via lower and upper solutions and variational methods," Journal of Mathematical Analysis and Applications, vol. 387, no. 2, pp. 475-489, 2012.

[30] D. Zhang and B. Dai, "Existence of solutions for nonlinear impulsive differential equations with Dirichlet boundary conditions," Mathematical and Computer Modelling, vol. 53, no. 5-6, pp. 1154-1161, 2011.

[31] H. Zhang and Z. Li, "Variational approach to impulsive differential equations with periodic boundary conditions," Nonlinear Analysis: Real World Applications, vol. 11, no. 1, pp. 67-78, 2010.

[32] B. Ricceri, "A further three critical points theorem," Nonlinear Analysis: Theory, Methods \& Applications, vol. 71, no. 9, pp. 41514157, 2009.

[33] B. Ricceri, "A three critical points theorem revisited," Nonlinear Analysis: Theory, Methods \& Applications, vol. 70, no. 9, pp. 3084-3089, 2009.

[34] B. Ricceri, "Existence of three solutions for a class of elliptic eigenvalue problems," Mathematical and Computer Modelling, vol. 32, no. 11-13, pp. 1485-1494, 2000.

[35] J. Mawhin and M. Willem, Critical Point Theory and Hamiltonian Systems, vol. 74, Springer, New York, NY, USA, 1989.
[36] J. Sun, H. Chen, and J. J. Nieto, "Infinitely many solutions for second-order Hamiltonian system with impulsive effects," Mathematical and Computer Modelling, vol. 54, no. 1-2, pp. 544555, 2011.

[37] H. Brezis and L. Nirenberg, "Remarks on finding critical points," Communications on Pure and Applied Mathematics, vol. 44, no. 8-9, pp. 939-963, 1991. 


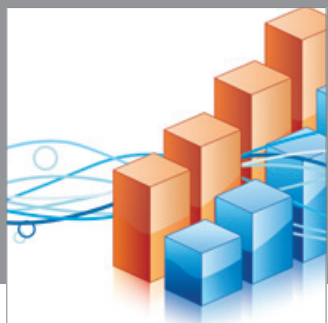

Advances in

Operations Research

mansans

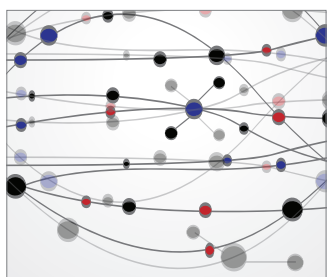

The Scientific World Journal
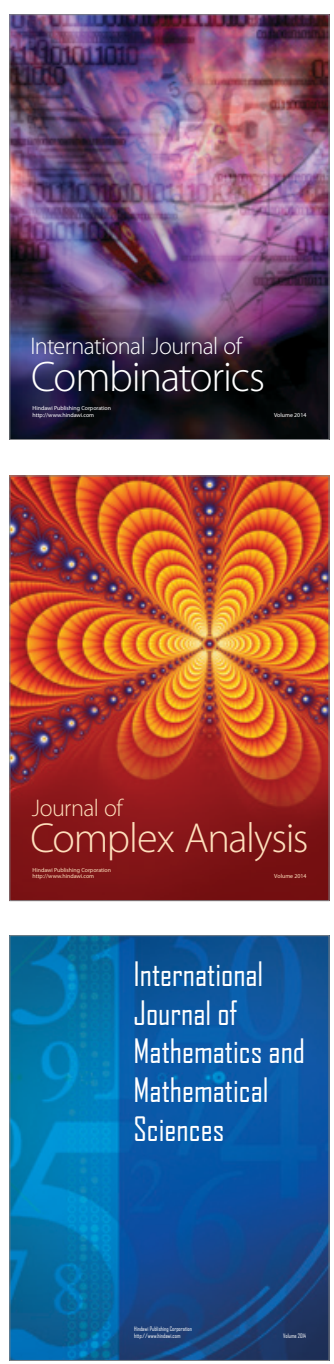
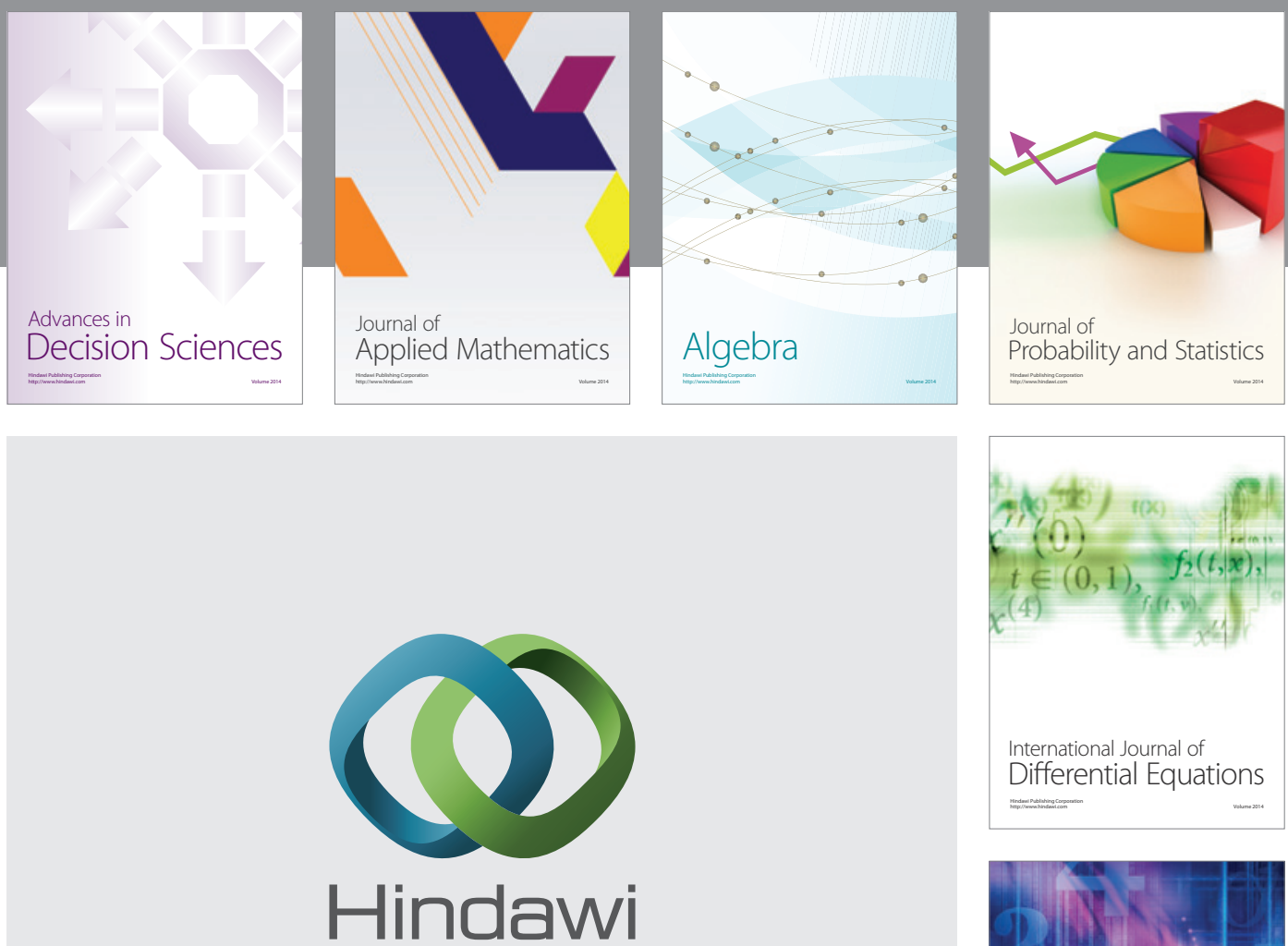

Submit your manuscripts at http://www.hindawi.com
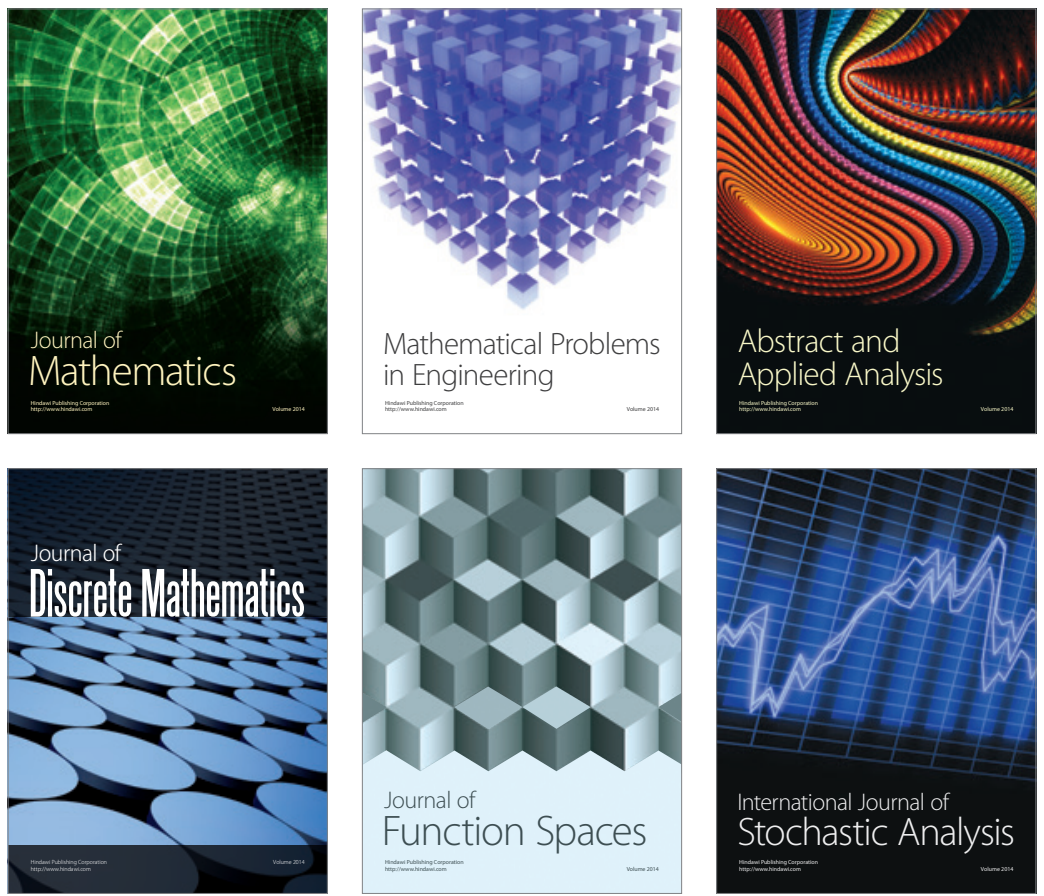

Journal of

Function Spaces

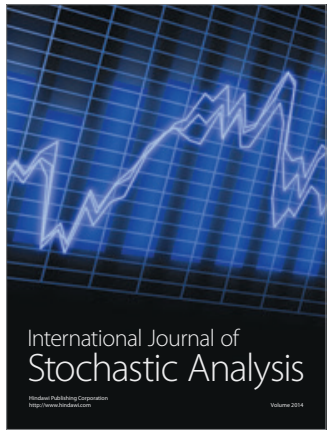

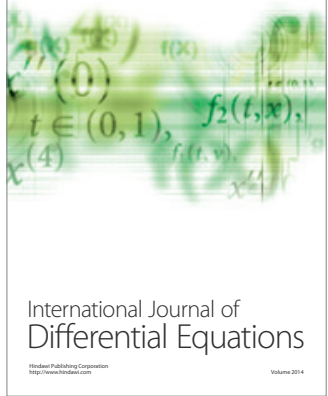
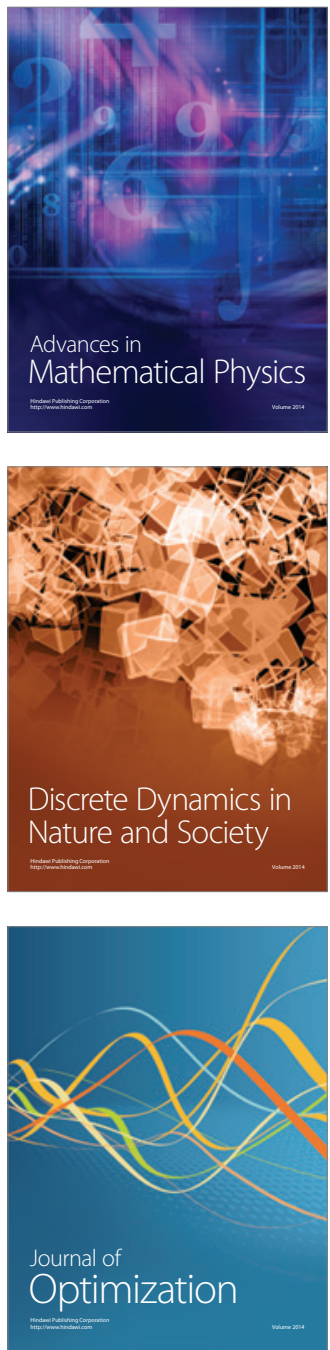